\title{
An Occult Leydig Cell Tumour in a Postmenopausal Woman Presenting with Alopecia and Hirsutism: A Case Report
}

\author{
Shuann Shwana, ${ }^{1}$ Natasha Shrikrishnapalasuriyar, ${ }^{1}$ Win Yin, $^{1}$ Monica Vij $^{2}$ and Atul Kalhan \\ 1. Royal Glamorgan Hospital, Llantrisant, Wales, UK; 2. Singleton Hospital, Swansea, Wales, UK
}

DOI: https://doi.org/10.17925/EE.2021.17.1.75

$\mathrm{P}$ rogressive hirsutism and moderate to severe male-pattern balding in women requires exclusion of an adrenal or ovarian tumour, especially in the presence of significantly elevated androgen levels. We present the case of a 68-year-old woman who was referred to an endocrinology clinic with insidious onset excessive facial hair growth and loss of scalp hair. Her testosterone levels were significantly elevated at $13 \mathrm{nmol} / \mathrm{L}$ (normal range: $0.1-1.4 \mathrm{nmol} / \mathrm{L}$ ), although dehydroepiandrosterone sulphate and 17-hydroxyprogesterone levels were normal, suggestive of an ovarian source of androgens. Repeated radiologic investigations, including pelvic ultrasound, and abdominal and pelvic computed tomography, could not identify the obvious source of androgens. Based on strong clinical suspicion of an ovarian tumour, she underwent an elective salpingo-oophorectomy, which detected an occult Leydig cell tumour on histopathological analysis. Post-operatively, her hyperandrogenic features significantly improved and testosterone levels normalized.

\section{Keywords}

Leydig cell tumour, hirsutism, testosterone

Disclosures: Shuann Shwana, Natasha

Shrikrishnapalasuriyar, Win Yin, Monica Vii and Atul

Kalhan have no financial or non-financial relationships or

activities to declare in relation to this article.

Acknowledgements: The authors wish to thank

Jason Shannon, Consultant Histopathologist,

who commented on the histology slides.

Review process: Double-blind peer review.

Compliance with ethics: Informed consent was received from the patient involved in this case study.

Authorship: The named authors meet the International Committee of Medical Journal Editors (ICMJE) criteria for authorship of this manuscript, take responsibility for the integrity of the work as a whole, and have given final approval for the version to be published.

Access: This article is freely accessible at

touchENDOCRINOLOGY.com. (C) Touch Medical Media 2021

Received: 23 June 2020

Accepted: 14 July 2020

Published online: 1 April 2021

Citation: touchREVIEWS in Endocrinology.

2021;17(1):75-8

Corresponding author: Prof. Atul Kalhan, Consultant Diabetes \& Endocrinology, Royal Glamorgan Hospital, Llantrisant, Wales, CF72 8TA, UK.

E: atul.kalhan@wales.nhs.uk

Support: No funding was received in the publication of this article.
Postmenopausal state is associated with changes in the hormonal milieu characterized by reduced oestrogen, increased gonadotrophins and a small increase in testosterone levels. ${ }^{12}$ As a result, it is not uncommon for women in postmenopausal age group to show mild increases in androgendriven hair growth. ${ }^{3,4}$ However, rapidly progressive hirsutism, acne and male-pattern alopecia in women warrants a search for an ovarian or adrenal androgen-secreting tumour. ${ }^{5}$ Androgensecreting ovarian tumours include Leydig cell tumours (LCTS), granulosa-theca cell tumours and gynandroblastoma tumours. ${ }^{5}$ LCTs are extremely rare accounting for $<0.1 \%$ of all ovarian tumours, ${ }^{6}$ with a majority of these tumours (95\%) being unilateral; however, radiological localization of these tumours may be challenging. 7.8

We present a case of a 68-year-old woman with rapidly progressive hirsutism and elevated androgen levels, in whom the primary source of androgen secretion remained occult despite multiple radiologic investigations. Based on strong clinical suspicion of an ovarian source of androgen secretion, she underwent an elective bilateral salpingo-oophorectomy which confirmed the diagnosis of LCT on histopathological analysis.

\section{Clinical presentation}

A 68-year-old White European woman was referred to the endocrinology clinic in view of rapidly progressive excessive facial and body hair growth, in addition to thinning of scalp hair. She had no associated fever, weight loss, anorexia, night sweats or systematic illness. Her background medical history included diverticular disease, although she was not on any regular medication. There was no history of exogenous testosterone exposure and no history of menstrual irregularity or hirsutism during her reproductive years. On general physical examination, her blood pressure was $110 / 70 \mathrm{mmHg}$. She had clinical features of significant hyperandrogenism, including frontal balding and facial and body hair growth. There was no proximal myopathy, easy bruisability or purple stria on abdomen suggestive of hypercortisolism.

Baseline investigations included full blood count and renal and liver function test. Electrolytes were within the normal range, although serum testosterone levels were significantly elevated at $13 \mathrm{nmol} / \mathrm{L}$ (normal range: $0.1-1.4 \mathrm{nmol} / \mathrm{L}$ ), which raised a strong clinical suspicion of an ovarian or adrenal androgen-secreting tumour. She had normal dehydroepiandrosterone sulphate (DHEA-S) and 17-hydroxyprogesterone (17-OHP) levels, which suggested a likely ovarian aetiology for raised androgen levels. Ovarian tumour-marker screening was negative. The detailed hormone profile during initial endocrinology clinic visit is shown in Table 1.

Despite such rapidly progressive androgenic features and significantly elevated testosterone levels, subsequent radiology, including an abdominal computed tomography (CT) scan and pelvic 
Table 1: Patient hormone profile at initial presentation to endocrinology clinic.

\begin{tabular}{|l|l|l|}
\hline Hormone & Patient levels & Normal range \\
\hline Follicle stimulating hormone, IU/L & 98.4 & $26-135$ \\
\hline Luteinizing hormone, IU/L & 34.2 & $7.7-59.0$ \\
\hline Oestradiol, pmol/L & $<18$ & $<183$ \\
\hline Testosterone, nmol/L & 13 & $0.1-1.4$ \\
\hline Sex hormone binding globulin, nmol/L & 86 & $17-125$ \\
\hline Free androgen Index, \% & 15.1 & $0.2-3.6$ \\
\hline 17-hydroxyprogesterone, nmol/L & 1.4 & $<2.6$ \\
\hline Dehydroepiandrosterone sulfate, $\mu \mathrm{mol} / \mathrm{L}$ & 1.3 & $0.3-6$ \\
\hline Thyroid-stimulating hormone, $\mathrm{mU} / \mathrm{L}$ & 0.48 & $0.27-4.20$ \\
\hline Prolactin, mU/L & 143 & $100-500$ \\
\hline
\end{tabular}

ultrasound, failed to localize any obvious adrenal or ovarian mass. On a follow-up visit to the endocrinology clinic, her symptoms of increasing facial and body hair had worsened, adding to her distress. The repeat testosterone levels were persistently high at $10.4 \mathrm{nmol} / \mathrm{L}$, although there was no further increase as compared to baseline level prior to first clinic visit. Repeat dedicated CT of the abdomen and pelvis revealed no evidence of any suspicious lesion or mass in the adrenal or ovarian gland.

In view of a strong clinical suspicion of an ovarian source for androgen secretion, and after an informed discussion with the patient and family, an elective bilateral salpingo-oophorectomy was carried out. The histology confirmed the diagnosis of an LCT (Figures 1-3) which had hitherto remained occult on multiple radiological investigations. Reassuringly, her hyper-androgenic state-induced symptoms showed a significant improvement within weeks of surgery and post-operative testosterone normalized.

\section{Discussion}

The evaluation of features of hirsutism, which includes development of androgen-driven hair growth on the upper lip, chin and body in postmenopausal women, remains challenging especially considering such changes are deemed as cosmetic and attributed to age-related alteration of hormonal milieu. ${ }^{9}$ The differential diagnoses of clinical androgen excess in postmenopausal woman include ovarian and adrenal tumours, Cushing's syndrome and ovarian hyperthecosis. ${ }^{9}$ This is in contrast to polycystic ovary syndrome and classic and non-classic congenital adrenal hyperplasia (CAH) which remain the commonest aetiologies leading to hirsutism in women of reproductive age. ${ }^{10}$

A noticeable and progressive increase in facial and body hair, male-pattern frontal balding and masculinization of the voice are a few of the key symptoms which warrant further endocrinological evaluation in postmenopausal women. This patient, who was aged 68 years at the time of initial presentation, had noticed hair growth on her upper lip and chin apart from abdomen, which she found socially distressing. It was significant to note that she had no history of menstrual irregularity and/or hirsutism features while she was in her reproductive years, making the possibility of polycystic ovary syndrome and CAH unlikely. There was no history of exogenous testosterone exposure which at times can induce mild to moderate increase in serum testosterone levels. It is important to obtain a detailed history of possible testosterone use in patients and/or their partner to exclude the possibility of iatrogenic hyperandrogenism.
Figure 1: Background ovarian stroma at the top with a well-defined unencapsulated tumour at the bottom.

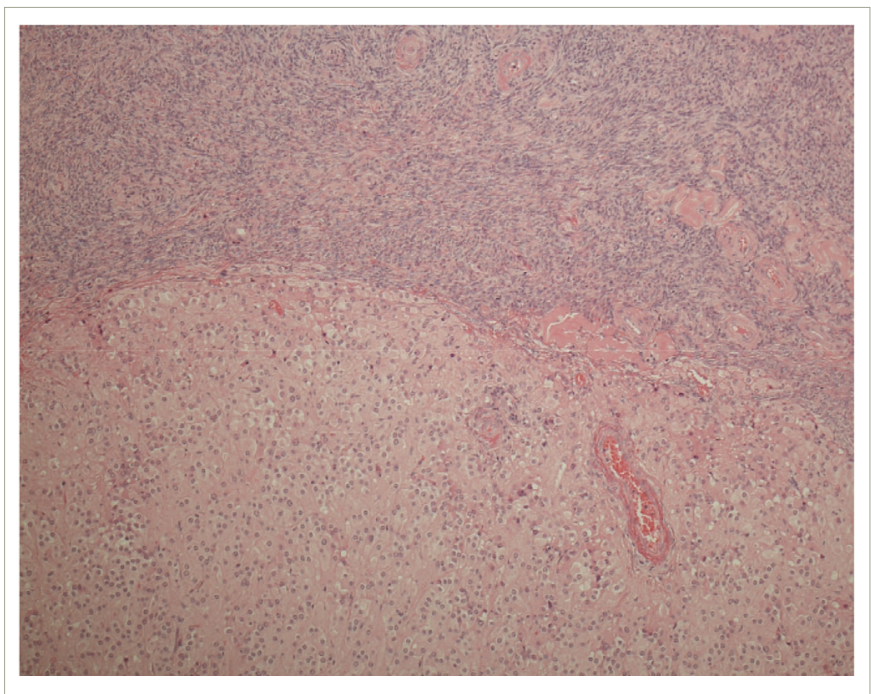

Figure 2: The tumour composed of nests of regular epithelioid cells with abundant eosinophilic cytoplasm and regular nuclei.

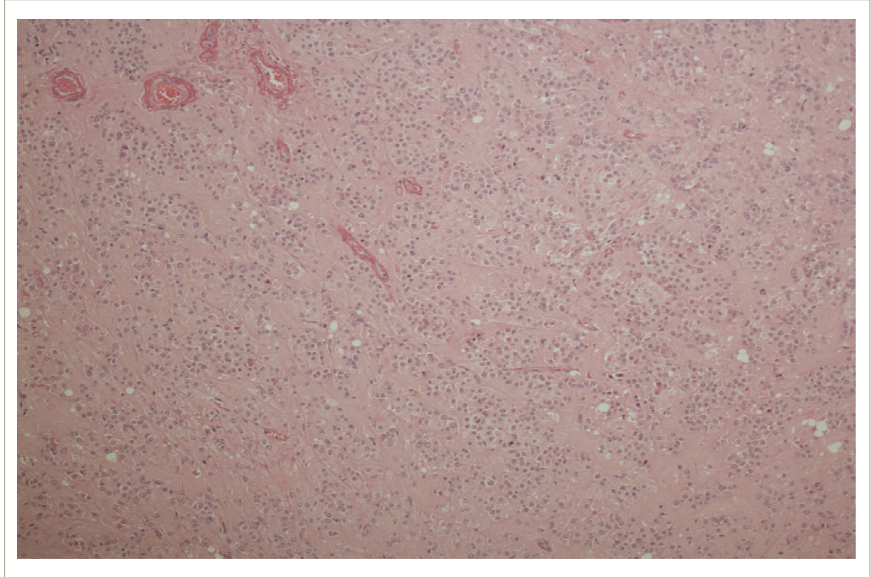

Figure 3: Cells magnified by x200 with evidence of characteristic cytoplasmic Reinke crystals (best seen as the rectangular pink fragments bottom right).

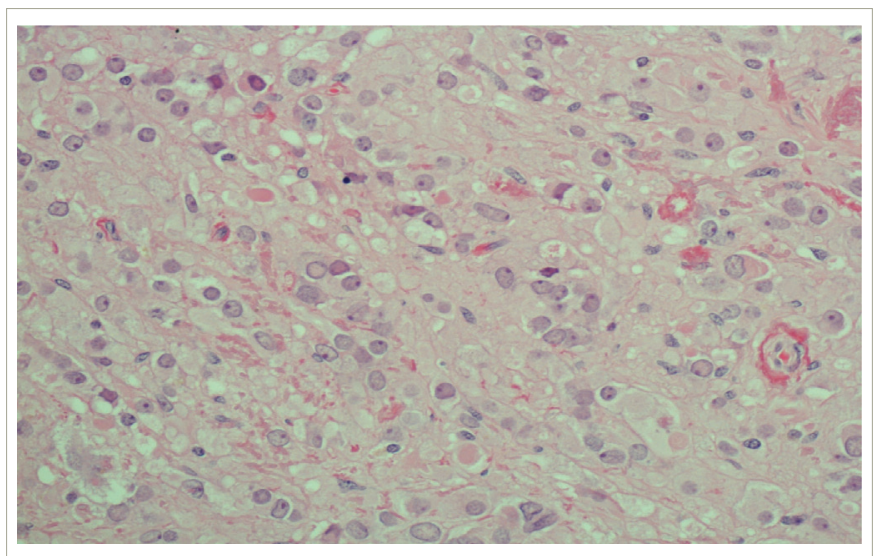


The clinical evaluation of women with rapidly progressive hyperandrogenic features includes gynaecological examination to assess presence of clitoromegaly or adnexal lesions. The initial baseline endocrinological investigation includes measurement of serum testosterone, DHEA-S, gonadotrophins, oestradiol and thyroid function test. ${ }^{\text {It }}$ is worth noting that under physiological states, circulating testosterone levels in women is $0.1-1.4 \mathrm{nmol} / \mathrm{L}$ with equal contribution from the ovaries as well as adrenal glands. ${ }^{11}$ Postmenopausal ovaries can be considered an androgen-secreting organ, especially with a steep decline in oestrogen levels. In contrast, DHEA-S is primarily produced in zona reticularis of adrenal cortex and remains under regulation of adrenocorticotrophic hormone. DHEA-S has a good sensitivity with a good negative predictive value, although its specificity (85-98\%) is deemed relatively low for identifying androgen secreting adrenal tumour. ${ }^{12}$

A significant rise in testosterone levels ( $>5 \mathrm{nmol} / \mathrm{L}$ ) and DHEA-S levels within normal range suggest ovarian tumour as the most likely source of excess androgens. In addition, although it is relatively rare for non-classic CAH to present at such a late stage of life, checking 17-OHP as a routine part of evaluation of occult hyperandrogenism remains a pragmatic approach, especially considering efficacy of steroid therapy in alleviating the signs and symptoms of hyperandrogenism in this condition. In our patient, initial testosterone levels were elevated (13.1 nmol/L) and DHEA-S as well as 17-OHP levels were within normal range which suggested high likelihood of an ovarian androgen-secreting tumour warranting further radiological investigations.

Transabdominal/transvaginal ultrasound, apart from abdominal CT, are usually the initial radiological modalities used to evaluate a possible ovarian source of excess androgens. ${ }^{5}$ Magnetic resonance imaging can be used in hyperandrogenic postmenopausal women with normal ultrasonography and has a $78 \%$ positive and $100 \%$ negative predictive value. 13,14 Alternatively, use of positron emission tomography (PET), with androgen-secreting ovarian tumours showing high uptake of ${ }^{18} \mathrm{~F}$-2-fluorodeoxy-D-glucose, can improve diagnostic sensitivity. ${ }^{15}$ In our patient, repeated pelvic ultrasound and abdominal CT failed to identify any obvious source of androgen excess. The lack of availability of PET scan in our district general hospital remained a limiting factor.

Combined ovarian and adrenal venous sampling has been proposed as a diagnostic modality to localize the occult source of androgens for postmenopausal women. ${ }^{16}$ However, the positive diagnostic yield of such an invasive procedure has been as low as $26-45 \%$, apart from the technical challenges involved in simultaneous catheterization of ovarian and adrenal veins. ${ }^{17}$ This diagnostic procedure perhaps should be reserved for women of reproductive age wishing to conceive so that the non-androgen-secreting ovary can be preserved. In our patient, we opted for elective bilateral oophorectomy as the next step in management, as preservation of ovarian function had limited functional implications.

The histopathological analysis of ovaries confirmed LCT as the diagnosis of androgen secretion in our patient. LCTs are rare $(<0.1 \%$ of ovarian sex cord tumours) and are mostly diagnosed in postmenopausal women. They are generally unilateral and benign. ${ }^{18,19}$ The diagnosis of LCT is confirmed by histopathology with inhibin and calretinin are the most useful immunohistochemical markers.
The management of androgen-secreting ovarian tumours is based on histology, stage of the disease and the patient's wishes to conserve fertility. Since LCTs are usually localized to the ovaries, bilateral oophorectomy should be considered as a preferred therapeutic option in women with clinically and biochemically significant hyperandrogenism after exclusion of adrenal tumour and $\mathrm{CAH}_{.}{ }^{20}$ In our patient, the hyperandrogenic symptoms and signs improved significantly after surgery and her repeat serum testosterone levels were $<0.1 \mathrm{nmol} / \mathrm{L}$, suggestive of complete excision of source of androgen. It is worth noting that a hyperandrogenic state is associated with increased low-density lipoprotein cholesterol and triglycerides, reduced high-density lipoprotein cholesterol, insulin resistance, raised haematocrit and increased risk of development of cardiovascular disease..$^{21,22}$ As a result, elective bilateral oophorectomy in a radiologically-occult androgen-secreting tumour (after exclusion of adrenal source) has potential to significantly improve long-term prognosis in such women.

\section{Take-home message}

Women with insidious-onset progressive hirsutism along with significantly raised serum testosterone levels ( $>5 \mathrm{nmol} / \mathrm{L}$ ) should be screened radiologically for ovarian or adrenal tumours. Radiologic localization of LCTs can be challenging considering their small size (mostly $<4 \mathrm{~cm}$ ) despite their unique radiologic characteristics (isoechoic on ultrasonography and isodense on CT scan). Selective ovarian sampling to localize the primary tumour remains an invasive procedure and has limited role, if any, in women who no longer wish to conceive and in whom elective bilateral oophorectomy is the preferred management option. $\square$

\section{Patient perspective}

At the start of my journey, I was a fairly fit 68-year-old lady. I have an underactive thyroid and high blood pressure, both controlled by medication. I have been married for 50 years and have three children: twin sons and a daughter. I had an early menopause, at 40 years of age.

While seeing my GP, I mentioned to her that my hair was thinning and I had noticed excessive hair growth on my chin and upper lip, so she said she would take a blood test to check my testosterone. Three days later, I received a phone call from my GP telling me that my testosterone level was very high and that I needed an urgent CT scan. Two weeks later nothing unusual appeared in my ovaries and adrenal glands, which was where the problem should have been, so was I told. I was referred to the endocrinology clinic, where I had further blood tests and further two scans over the next 3-4 months. All came back showing nothing unusual except an area of inflammation in my bowel. A colonoscopy was performed and diverticula disease diagnosed. I also had an internal and external ultrasound taken with nothing showing up. Further blood tests showed testosterone levels were still high. It was recommended, by my endocrinologist, that I should have my ovaries and fallopian tubes removed, which I was able to have done privately in October 2019

This resulted in the discovery of the tumour. I will be checked every 4 months for the first year then every 6 months the next year. During all my treatment, I felt perfectly fit and well and my testosterone level is almost nil. My hair growth on my chin has definitely reduced and my hair is slowly thickening. 
1. Morely JM, Perry HM. Androgens and women at the menopause and beyond I Gerontol A Biol SCi Med SCi. 2003:58:M409-16.

2. Burger $\mathrm{HG}$, Dudley $\mathrm{EC}$, Cui J, et al. A prospective longitudinal study of serum testosterone, dehydroepiandrosterone sulfate, and sex hormone-binding globulin levels through the menopause transition. J Clin Endocrinol Metab. 2000;85:2832-8

3. Atmaca M, Seven I, Üçler R, et al. An interesting cause of hyperandrogenemic hirsutism. Case Rep Endocrinol 2014;2014:987272

4. Gandrapu B, Sundar P, Phillips B. Hyperandrogenism in a postmenopausal woman secondary to testosterone secreting ovarian stromal tumor with acoustic schwannoma. Case Rep Endocrinol. 2018;2018:8154513.

5. Alpanes M, Gonzalez-Casbas JM, Sanchez J, et al. Management of postmenopausal virilization. J Clin Endocrinol Metab. 2012;97:2584-8

6. Stegner HE, Loning T. [Endocrine-active tumors of the ovary]. Pathologe. 2003;24:314-22

7. Kaltsas GA, Isidori AM, Kola BP, et al. The value of the low-dose dexamethasone suppression test in the differential diagnosis of hyperandrogenism in women. J Clin Endocrinol Metab. 2003;88:2634-43.

8. Kaltsas GA, Mukherjee JJ, Kola B, et al. Is ovarian and adrenal venous catheterization and sampling helpful in the investigation of hyperandrogenic women? Clin Endocrinol
(Oxf). 2003;59:34-43

9. Rothman MS, Wierman ME. How should postmenopausa androgen excess be evaluated? Clin Endocrinol (Oxf). 2011;75:160-4.

10. Martin KA Anderson RR, Chang RJ, et al. Evaluation and treatment of hirsutism in premenopausal women: an endocrin society clinical practice guideline. J Clin Endocrinol Metab. 2018;103:1233-57.

11. Burger HG. Androgen production in women. Fertil Steril. 2002;77(Suppl. 4):S3-5

12. Hofland M, Cosyns S, De Sutter P, et al. Leydig cell hyperplasia and Leydig cell tumour in postmenopausal women: report of two cases. Gynecol Endocrinol. 2013;29:213-5.

13. Sarfati J, Bachelot A, Coussieu C, et al. Study Group Hyperandrogenism in Postmenopausal Women. Impact of clinical, hormonal, radiological, and immunohistochemical studies on the diagnosis of postmenopausal hyperandrogenism. Eur J Endocrinol. 2011;165:779-88. 14. Yance VRV, Marcondes JAM, Rocha MP, et al. Discriminating between virilizing ovary tumors and ovary hyperthecosis in image studies. Eur J Endocrinol. 2017:177:93-102.

15. Mattsson C, Stanhope CR, Sam S, et al. Image in endocrinology: testosterone-secreting ovarian tumor localized with fluorine18-2-deoxyglucose positron emission tomography. J Clin Endocrinol Metab. 2006;91:738-9.

16. Dickerson RD, Putman MJ, Black ME, Pinto KR, Diamond NG,
Marynick S, Pinto AB. Selective ovarian vein sampling to Iocalize a Leydig cell tumor. Fertil Steril. 2005;84:218.

17. Souto SB, Baptista PV, Braga DC, Carvalho D. Ovarian Leydig cell tumor in a post-menopausal patient with severe hyperandrogenism. Arq Bras Endocrinol Metabol. 2014;58:68-75.

18. Chen M, Zhou W, Zhang Z, et al. An ovarian Leydig cell tumo of ultrasound negative in a postmenopausal woman with hirsutism and hyperandrogenism. Medicine (Baltimore). 2018;97:e0093.

19. Nardo LG, Ray DW, Laing I, et al. Ovarian Leydig cell tumor in a peri-menopausal woman with severe hyperandrogenism and virilization. Gynecol Endocrinol. 2005;21:238-41.

20. Hofland M, Cosyns S, De Sutter P, et al. Leydig cell hyperplasia and Leydig cell tumour in postmenopausal women: report of two cases. Gynecol Endocrinol. 2013;29:213-5.

21. Wild RA, Carmina E, Diamanti-Kandarakis E, et al. Assessment of cardiovascular risk and prevention of cardiovascular disease in women with the polycystic ovary syndrome: a consensus statement by the Androgen Excess and Polycystic Ovary Syndrome (AE-PCOS) Society. J Clin Endocrinol Metab. 2010;95:2038-49.

22. Yetkin, D.O, Demirsoy, E.T. \& Kadioglu, P. Pure leydig cell tumour of the ovary in a post-menopausal patient with severe hyperandrogenism and erythrocytosis. Gynecol Endocrinol. 2011;27:237-40 